\title{
Gene Expression Profile Signature of Aggressive Waldenström Macroglobulinemia with Chromosome 6q Deletion
}

\author{
Naohiro Sekiguchi $\mathbb{D}^{\mathrm{D}},{ }^{1}$ Junko Nomoto, ${ }^{2}$ Akihisa Nagata, ${ }^{1}$ Masahiro Kiyota, ${ }^{1}$ \\ Ichiro Fukuda, ${ }^{3}$ Kazuaki Yamada, ${ }^{4}$ Naoki Takezako, ${ }^{1}$ and Yukio Kobayashi ${ }^{2}{ }^{2}$ \\ ${ }^{1}$ Division of Hematology, National Hospital Organization Disaster Medical Center, Tachikawa, Tokyo 190-0014, Japan \\ ${ }^{2}$ Department of Hematology, National Cancer Center Hospital, Tsukiji, Tokyo 104-0045, Japan \\ ${ }^{3}$ Division of Radiology, National Hospital Organization Disaster Medical Center, Tachikawa, Tokyo 190-0014, Japan \\ ${ }^{4}$ Division of Laboratory and Pathology, National Hospital Organization Disaster Medical Center, Tachikawa, Tokyo 190-0014, Japan
}

Correspondence should be addressed to Yukio Kobayashi; ykkobaya@iuhw.ac.jp

Received 23 March 2018; Revised 21 August 2018; Accepted 10 September 2018; Published 4 October 2018

Academic Editor: Maria L. Tornesello

Copyright (C) 2018 Naohiro Sekiguchi et al. This is an open access article distributed under the Creative Commons Attribution License, which permits unrestricted use, distribution, and reproduction in any medium, provided the original work is properly cited.

\begin{abstract}
Background. Waldenström macroglobulinemia (WM) is a rare, indolent B-cell lymphoma. Clinically, chromosome 6q deletion ( $6 \mathrm{q}$ del) including loss of the B lymphocyte-induced maturation protein 1 gene (BLIMP-1) is reported to be associated with poor prognosis. However, it remains unclear how the underlying biological mechanism contributes to the aggressiveness of WM with 6q del. Methods. Here, we conducted oligonucleotide microarray analysis to clarify the differences in gene expression between WM with and without 6q del. Gene ontology (GO) analysis was performed to identify the main pathways underlying differences in gene expression. Eight bone marrow formalin-fixed paraffin-embedded samples of WM were processed for interphase fluorescence in situ hybridization analysis, and three were shown to have 6q del. Results. GO analysis revealed significant terms including "lymphocyte activation" (corrected p value=6.68E-11), which included 31 probes. Moreover, IL21R and JAK3 expression upregulation and activation of the B-cell receptor signaling (BCR) pathway including CD79a, SYK, BLNK, PLC 2 , and CARD11 were detected in WM with 6q del compared with WM without 6q del. Conclusion. The present study suggested that the BCR signaling pathway and IL21R expression are activated in WM with 6q del. Moreover, FOXP1 and CBLB appear to act as positive regulators of the BCR signaling pathway. These findings might be attributed to the aggressiveness of the WM with 6q del expression signature.
\end{abstract}

\section{Background}

Waldenström macroglobulinemia (WM) is a rare, indolent type of B-cell lymphoma with monoclonal IgM gammopathy. B-cells, lymphoplasmacytic cells, and plasma cells (PCs) form neoplasms in WM, with the bone marrow (BM) being the main site of infiltration [1]. The disease has an overall annual incidence of three per million in the United States (US) $[2,3]$.

Cytogenetic analysis previously detected chromosome 6q deletion (del), including loss of the B-lymphocyte-induced maturation protein 1 gene (BLIMP-1) and TNFAIP3/A20, in $40 \%-55 \%$ of WM patients in the US and European countries [4-8]. Furthermore, this aberration is recognized as one of the factors associated with poor prognosis, higher serum IgM levels, and a higher International Prognostic Scoring System for WM (ISSWM) level [4-8].

However, it remains unclear how the underlying biological mechanism contributes to the observed aggressiveness of WM with $6 \mathrm{q}$ del. The present study therefore conducted an oligonucleotide microarray analysis to clarify the biological differences between WM with and without $6 \mathrm{q}$ del.

\section{Methods}

2.1. Patients. Newly diagnosed symptomatic WM patients attending the National Hospital Organization Disaster Medical Center from January 2010 to March 2014 were enrolled in this study. Samples of cases whose comprehensive agreement 
had been obtained were subject for the study. The WM diagnostic criteria used in the present study were according to the revised $4^{\text {th }}$ edition of World Health Organization (WHO) classification of tumours of haematopoietic and lymphoid tissues [1]. Briefly, these included detection of the IgM monoclonal protein and BM neoplastic cells including lymphocytes, CD20-positive lymphoplasmacytic cells, and some PCs more than $10 \%$ and presence of symptoms.

Patient clinical data were obtained from their medical charts and included age, sex, performance status (PS), hemoglobin level, platelet count, serum $\beta 2$-microglobulin level, ISSWM [9], serum M-protein, B symptoms, hepatomegaly, splenomegaly, and lymphadenopathy. PS was determined according to the Eastern Cooperative Oncology Group scale [10].

2.2. Pathological Review. A pathological review confirmed that all cases were IgM-WM according to the revised 4th edition of WHO classification [1]. MALT lymphoma or CLL with monoclonal IgM protein were carefully excluded from the study. Using BM biopsied specimens, the infiltration pattern of neoplastic cells was divided into four groups: diffuse, interstitial, paratrabecular, and nodular. Additionally, the PC\%, lymphoplasmacytic cell \%, lymphocyte \%, and total neoplastic cells\% in BM were evaluated from BM smears.

2.3. Fluorescence In Situ Hybridization Analyses (FISH). Cytogenetic aberrations were obtained by FISH analysis using the A20/BLIMP-1/SHGC-79576 Three Color Probe (Cancer Genetics Italia $^{\mathrm{TM}}$ SRI, Mila, Italy) to detect $6 \mathrm{q}$ del. $A 20$ and BLIMP-1 are located at $6 \mathrm{q} 23$ and $6 \mathrm{q} 21$, respectively, and the $6 \mathrm{q}$ deletion can be easily detected by comparing the signal intensity of this region with that of the chromosome 6 centromere [11]. Interphase FISH was performed using stored BM aspirate patient specimens, and results were recorded according to ISCN 2016 [12]. The normal cut-off values for deletions in the chromosomal region were defined as means $+3 \mathrm{SD}$ of the results for 20 normal controls.

2.4. Oligonucleotide Microarray Analyses. cDNA microarray analysis was performed following the manufacture's instruction. Briefly, formalin-fixed paraffin-embedded BM biopsied samples were deparaffinized and total RNA was extracted using the RNeasy FFPE kit (Qiagen, Venlo, the Netherlands). Isolated total RNA was converted into cDNA, followed by cDNA amplification. After this, $4 \mu \mathrm{g}$ of cDNA was fragmented at $37^{\circ} \mathrm{C}$ for $30 \mathrm{~min}$ and then biotinylated using the Encore cDNA (Nugen, San Carlos, CA). Biotin-labeled cDNA was hybridized to the Human Genome U133 Plus 2.0 Array (Affymetrix, Santa Clara, CA). The array was incubated for 18 $\mathrm{h}$ at $45^{\circ} \mathrm{C}$, then automatically washed, and stained using the GeneChip Hybridization, Wash, and Stain Kit (Affymetrix). The array was scanned using a GeneChip Scanner 3000 7G.

2.5. Immunohistochemistry. Paraffin-embedded BM specimens underwent heat-induced antigen retrieval in $\mathrm{pH} 6.0$ citrate buffer. Polyclonal antibodies against IL-21R (1:500, NBP1-87502, Novus Biological, Littleton, CO) were used.
After incubation with the primary antibody, slides were incubated with the secondary antibody, One-Step PolymerHRP (BioGenex, Fremont, CA), for visualization. The IL-21R staining pattern was categorized into three groups: $<30 \%$, $30 \%-70 \%$, and $>70 \%$ of lymphoplasmacytic cells and PCs.

2.6. Statistical Analysis. Descriptive statistics of each clinical data were calculated by IBM SPSS Statistics for Windows 24.0J. Oligonucleotide microarray analyses were carried out using GeneSpring GX software version 13.0 (Agilent Technologies, Santa Clara, CA). Briefly, described as follows, the expression values were calculated from the raw data using the MBEI algorithm [13]. Only probes that signal intensity were within 20 to 100 percentile in each array which were included in the comparative analysis. The genes corresponding to probes having a change in intensity exceeding a ratio of four were considered as genes with a significant differential expression pattern. In parallel, unpaired t-test with unequal variance (Welch's t-test) was performed to compare the means of the two groups of replicates. Probes with a $\mathrm{p}$ value less than 0.05 were considered having significant different signal value. GO analysis was then performed on the sets of probes above the 4 -fold ratio that passed the $t$-test to identify the main pathways underlying differences in gene expression. We arbitrary selected 4-fold different expression as a cut-off ratio instead of considering false discovery rate, because of the small sample size, and we performed the oligonucleotide microarray analysis as an exploratory research. Ontologies with corrected $\mathrm{p}$-values $<0.1$ were considered significant. The microarray datasets used in the present study were deposited in the Gene Expression Omnibus under accession number GSE70511 (http://www.ncbi.nlm.nih.gov/geo/ query/acc.cgi?acc=GSE70511).

\section{Results}

3.1. Clinical Features. Eight patients were enrolled in the study, and characteristics are shown in Table 1. Briefly, the median age was 71.5 years, and the male/female ratio was $5 / 3$. ISSWM low/int/high was observed as $0 / 4 / 4$. Median monoclonal M-protein levels were $2.65 \mathrm{~g} / \mathrm{dL}$ (range: $0.8-4.28$ $\mathrm{g} / \mathrm{dL}$ ). A total of two patients were observed to have constitutional B symptoms; one had hepatomegaly, and another had splenomegaly. Adenopathy and cold agglutinin disease were found in two patients each. Diffuse, interstitial, paratrabecular, and nodular patterns of neoplastic cell invasion into the BM were seen in seven, zero, one, and zero patients, respectively. The median small lymphocyte $\%$, lymphoplasmacytic cell \%, and PC\% were $39.0 \%, 3.3 \%$, and $1.9 \%$, respectively, with total neoplastic cells accounting for $45.7 \%$.

3.2. Cytogenetic Analysis and Correlations between $6 q$ del and Individual Characteristics. To detect $6 \mathrm{q}$ del, fluorescent in situ hybridization (FISH) analysis was performed (Figures 1(a) and 1(b)). The cut-off value for loss of BLIMP-1 and A20 was $3 \%$. Three of the eight cases had 6q del, in which both genes were deleted, and the other five cases did not have $6 \mathrm{q}$ del. Median serum M-protein levels were $3.14 \mathrm{~g} / \mathrm{dL}$ in patients with $6 \mathrm{q}$ del, compared with $2.25 \mathrm{~g} / \mathrm{dL}$ in those without $6 \mathrm{q}$ 
TABLE 1: Patient characteristics.

\begin{tabular}{|c|c|c|c|}
\hline & all $(\mathrm{N}=8)$ & $6 \mathrm{q}$ del $(+)(\mathrm{N}=3)$ & $6 \mathrm{q}$ del $(-)(\mathrm{N}=5)$ \\
\hline Median Age (old, range) & $71.5(58-79)$ & $76(70-79)$ & $71(58-76)$ \\
\hline $\operatorname{Sex}(M / F)(\%)$ & $5 / 3$ & $2 / 1$ & $3 / 2$ \\
\hline PS $>1(\%)$ & 5 & 2 & 3 \\
\hline $\mathrm{Hb}<11.5 \mathrm{~g} / \mathrm{dL}(\%)$ & 6 & 3 & 3 \\
\hline plt $<10 \times 10^{9} / \mathrm{L}(\%)$ & 1 & 0 & 1 \\
\hline$\beta 2 \mathrm{MG}>3 \mathrm{mg} / \mathrm{L}(\%)$ & $3(64.3)$ & 2 & 2 \\
\hline Median M-protein (g/dL) & $2.65(0.8-4.28)$ & $3.14(2.13-4.28)$ & $2.25(0.8-3.87)$ \\
\hline ISSWM(Low/Int/High) (\%) & $0 / 4 / 4(9 / 36 / 55)$ & $0 / 1 / 2$ & $0 / 3 / 2$ \\
\hline B-symptom (\%) & $2(29.4)$ & 1 & 1 \\
\hline Hepatomegaly (\%) & $1(23.8)$ & 0 & 1 \\
\hline Splenomegaly (\%) & $1(23.8)$ & 0 & 1 \\
\hline Lymphadenopathy (\%) & $2(25)$ & 0 & 2 \\
\hline Cold agglutinin disease & 2 & 0 & 2 \\
\hline Infiltration pattern $(\mathrm{D} / \mathrm{P})$ & $7 / 1$ & $3 / 0$ & $4 / 1$ \\
\hline Plasma cell (\%) & $1.9(1-3.6)$ & $2.0(1-2.2)$ & $1.8(1.2-3.6)$ \\
\hline Lymphoplasmacytic cell (\%) & $3.3(2.4-8.6)$ & $7.1(2.6-8.6)$ & $3.0(2.4-5.2)$ \\
\hline Small lymphocyte (\%) & $39.0(29.4-62.8)$ & $41.6(35.9-62.8)$ & $36.4(29.4-46.8)$ \\
\hline Total neoplastic cell (\%) & $45.7(36-72.4)$ & $46.4(45-72.4)$ & $41.4(36-50.4)$ \\
\hline
\end{tabular}

PS: performance status; Hb: hemoglobin; plt: platelets; $\beta 2 \mathrm{MG}$ : $\beta 2$-microglobulin; cCa: calculated Ca; ISSWM: International Prognostic Scoring System for Waldenström Macroglobulinemia.

del. ISSWM low/int/high was $0 / 1 / 2$ in patients with $6 \mathrm{q}$ del, compared with $0 / 3 / 2$ in patients without $6 q$ del. The median small lymphocyte \%, lymphoplasmacytic cell \%, PC \%, and total neoplastic cell \% were $41.6 \%, 7.1 \%, 2.0 \%$, and $46.4 \%$, respectively, in those with $6 \mathrm{q}$ del, versus $36.4 \%, 3.0 \%, 1.8 \%$, and $41.4 \%$, respectively, in those without $6 \mathrm{q}$ del.

3.3. Oligonucleotide Microarray. All the eight cases' samples were subjected for oligonucleotide microarray analysis. To clarify the differences in gene expression between WM with and without $6 \mathrm{q}$ del, we performed oligonucleotide microarray analyses and detected that a total of 428 probes, corresponding to 324 annotated genes, were upregulated ( $p$ value $<0.05$ and fold change $>4.0$ ), and a total of 112 probes, corresponding to 96 annotated genes, were downregulated ( $\mathrm{p}$ value $<0.05$ and fold change $<0.25$ ) in WM with $6 \mathrm{q}$ del (Supplementary Tables 1 and 2). Among genes located on chr 6q, 4 genes were upregulated, and 5 genes were downregulated, while both BLIMP-1 and A20 were neither upregulated nor downregulated.

Consecutively, gene ontology (GO) analysis was performed, and GO terms including "lymphocyte activation" (corrected $\mathrm{p}$ value $=6.68 \mathrm{E}-11$ ) and "B-cell activation" (corrected $p$ value $=2.15 \mathrm{E}-08$ ) were statistically significant in the upregulated gene lists relative to the all genes on the microarray (Table 2). A network analysis of GO terms associated with "lymphocyte activation" included 31 probes and 30 genes, listed in Table 3. Genes involved in the B-cell receptor (BCR) signaling pathway, including $C D 79 a, S Y K, B L N K, P L C \gamma 2$, and $C A R D 11$, were shown to be activated in WM with $6 \mathrm{q}$ del compared with WM without $6 \mathrm{q}$ del. Other upregulated genes included $I L-21 R, J A K 3, I F N \gamma$, and FOXP1. In contrast, the
GO term "plasma cell differentiation" was not statistically significant (data not shown).

3.4. Immunohistochemistry of Interleukin (IL)-21R. To confirm the upregulation of the IL21R protein in WM with $6 \mathrm{q}$ del, we performed immunostaining analysis. Figures $1(\mathrm{c})$ and $1(\mathrm{~d})$ show that all three patients with $6 \mathrm{q}$ del had $>70 \%$ positivity of IL-21R in lymphoplasmacytic cells and PCs. In WM without $6 \mathrm{q}$ del, one case was categorized as $30 \%-70 \%$ positivity, while the remaining four cases showed $<30 \%$ positivity.

\section{Discussion}

WM is a rare entity of low-grade B-cell lymphoma with IgM monoclonal gammopathy according to classification by the WHO [1]. The most commonly involved site of infiltration is the BM, and some patients have adenopathy, hepatomegaly, and splenomegaly [1]. Neoplastic cells consist of CD20positive B-cells and lymphoplasmacytic cells, while some PCs are also considered to be neoplastic [14]. Recently, Treon et al. [15] reported that the MYD88 L265P mutation has been recognized in about $90 \%$ of WM cases in the US and in $70 \%-90 \%$ of cases worldwide [16]. This mutation is also found in $47 \%$ of patients with IgM monoclonal gammopathy of undetermined significance (IgM-MGUS) and is associated with a higher risk of disease progression to WM and splenic marginal zone lymphoma [17]. It is known to be a major mechanism of oncogenesis and is linked with Toll-like receptor (TLR) signaling pathway activation [15]. Furthermore, Yang et al. identified Bruton tyrosine kinase (BTK) complexed to MYD88 in L265P-expressing WM cells, with preferential binding of MYD88 to phosphorylated BTK [18]. 


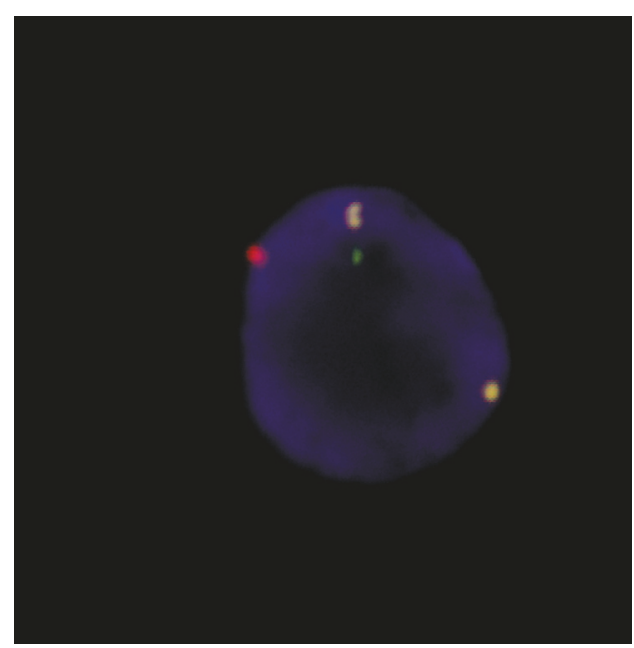

(a)

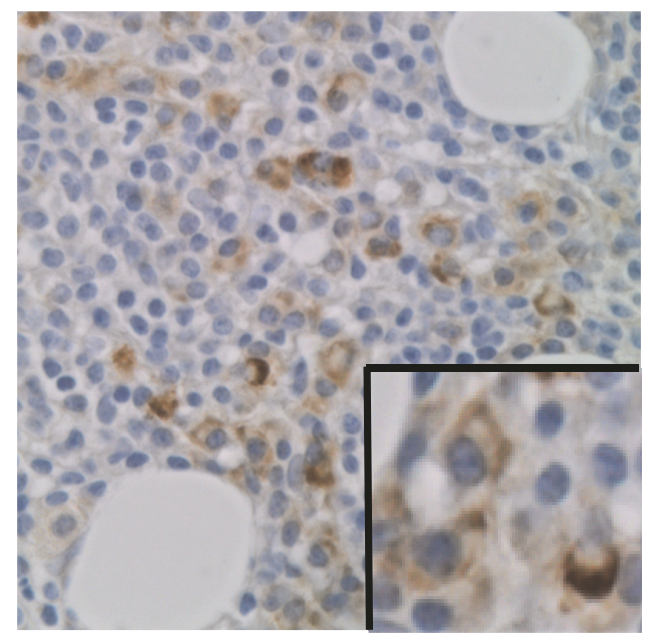

(c)

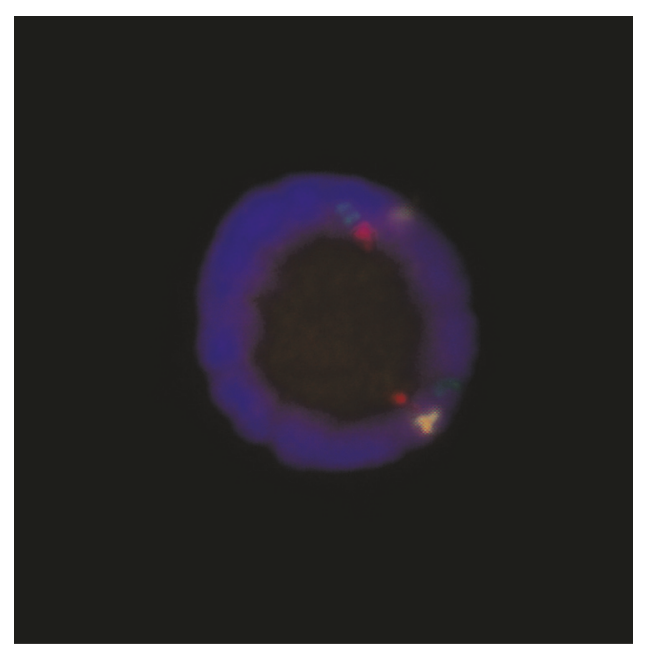

(b)

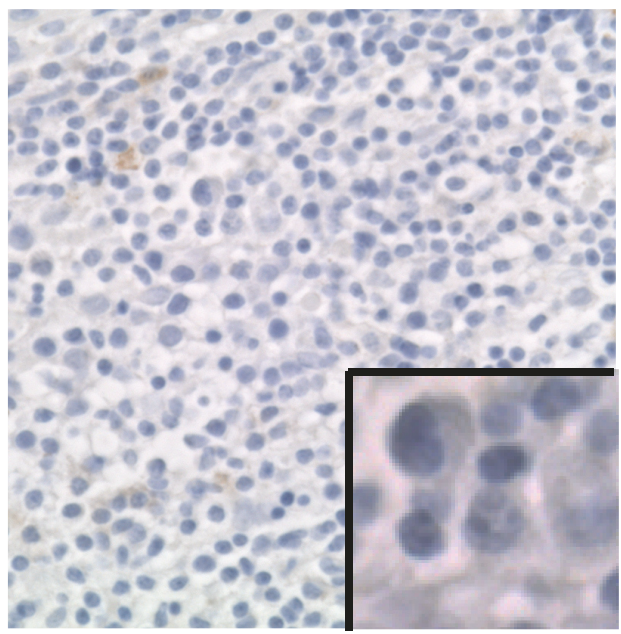

(d)

FIGURE 1: FISH analysis of BM aspirates using the A20/BLIMP1/SHGC-79576 Three Color Probe (Cancer Genetics Italia ${ }^{\mathrm{TM}}$ ) and IL21R staining of a BM biopsied specimen. Green, red, and yellow signals represent BLIMP1, A20, and SHGC-79576, respectively. (a) 6q del pattern. One green, one red, and two yellow signals were detected, showing nuc ish(SHGC-79576 x2, BLIMP-1x1, A20 x1). (b) Normal pattern. Two green, two red, and two yellow signals are recognized, showing nuc ish (SHGC-79576, BLIMP-1, A20) x2. (c) >70\% positivity of IL-21R in lymphoplasmacytic cells and plasma cells (original magnification $\times 600$ ). (d) $<30 \%$ positivity of IL-21R in lymphoplasmacytic cells and plasma cells (original magnification $\times 600$ ).

In contrast, 6q del, including loss of BLIMP-1 [19-21] and A20 [22, 23], is recognized in $40 \%-55 \%$ of WM cases in the US and European countries $[4-8,14]$. However, it is not recognized in IgM-MGUS, suggesting that it is a secondary event [7]. 6q del has long been thought to be involved in oncogenesis and is also reported to be associated with poor prognostic factors, such as higher levels of IgM and C-reactive protein and poor-risk patients with high ISSWM levels [4-6]. Nevertheless, the underlying biological mechanism of $6 \mathrm{q}$ del in WM is unclear.

Several studies regarding genes expression analyses in WM were performed, and these studies contributed to elucidate the biology and activated pathway in WM [24-26]. Chng et al. firstly conducted genes expression analyses in WM [24]. They reported that the most significantly upregulated gene was IL6, and the most significantly associated pathway for genes-set was MAPK signaling in WM compared to chronic lymphocytic leukemia (CLL) and multiple myeloma (MM). San Miguel and colleagues found deregulation of genes involved in plasma cell differentiation including $P A X 5$, which was overexpressed, while BLIMP-1 and IRF4 were underexpressed in WM in comparison with CLL and MM. In addition, they hypothesized that lack of $P A X 5$ repression contributed to the upregulation of 3 genes of BCR signaling pathway including CD79, BLNK, and $S Y K$ in WM [25]. Jiménez et al. reported that CD79A (B cell activation), IRF3, MYD88, MEK1, P38 (TLR pathway), and WNK1 (MAPK pathway) were overexpressed in WM compared to IgMMGUS, in which pathways might be responsible for WM cell growth and survival [26]. 
TABLE 2: Significant terms on the gene ontology list.

\begin{tabular}{lclc}
\hline GO Number & GO term & P-value & Numbers of probes \\
\hline GO:0046649 & lymphocyte activation & $6.68 \mathrm{E}-11$ & 31 \\
\hline GO:0043486 & histone exchange & $1.81 \mathrm{E}-10$ & 14 \\
\hline GO:0034508 & centromere complex assembly & $3.22 \mathrm{E}-10$ & 14 \\
\hline GO:0043044 & ATP-dependent chromatin remodeling & $1.02 \mathrm{E}-09$ & 14 \\
\hline GO:0045321 & leukocyte activation & $2.49 \mathrm{E}-09$ & 32 \\
\hline GO:1903706 & regulation of hemopoiesis & $9.44 \mathrm{E}-09$ & 27 \\
\hline GO:0042113 & B cell activation & $2.15 \mathrm{E}-08$ & 18 \\
\hline
\end{tabular}

GO, gene ontology.

TABLE 3: Genes up-regulated in WM with 6q del compared with WM without 6q del.

\begin{tabular}{|c|c|}
\hline Function categories & Up-regulated gene \\
\hline B-cell receptor signaling pathway & $C D 79 a, S Y K, B L N K, P L C \gamma 2, C A R D 11$ \\
\hline IL21/21R signaling pathway & $I L 21 R, J A K 3$ \\
\hline NF-kB activater & FOXP1 \\
\hline Ubiqutin ligase & $C B L B$ \\
\hline Ikaros zinc finger family & IFZF3 \\
\hline Cytokines & $I F N \gamma$ \\
\hline Other genes & $\begin{array}{l}\text { MSH6, IMPDH2, AKAP17A, CCR7, MEF2C, POU2F2, ITPKB, BANK1, IL7R, LAX1, } \\
\text { ERCC1, PRKCB, KLRC4-KLRK, HDAC9, ITGAL, GON4L, PSEN1, RHOH, PSEN1 }\end{array}$ \\
\hline
\end{tabular}

WM, Waldenstrom macroglobulinemia.

One of the unique findings of the present study was that genes involved in the BCR signaling pathway [23], including $C D 79 a, S Y K, B L N K, P L C \gamma 2$, and CARD11 are upregulated in WM with $6 \mathrm{q}$ del patients compared with those without $6 \mathrm{q}$ del. Little is known about BCR pathway upregulation in WM [25], although various subtypes of B-cell lymphomas are associated with BCR pathway activation [27]. However, Argyropoulos et al. suggested that the BCR pathway is activated in WM following phosphoprofiling analysis [28]. Moreover, it is widely accepted that BLIMP-1 suppresses B-cell proliferation and activation, including the BCR signaling pathway, and orchestrates mature PC differentiation by suppressing the expression of genes necessary for commitment and maintenance of the B-cell identity, including $P A X 5$ and $X B P-1[29,30]$.

During preparation for this manuscript, Staudt and colleague reported that MYD88, TLR9, and BCR complexes (My-T-BCR Complexes) exist in activated B-cell like diffuse large B-cell lymphoma and in WM, which might play a role in tumor growth and survival [31] (Figure 2). In the present study, the BCR signaling pathway was activated in WM with $6 \mathrm{q}$ del patients, which might result from the decreased inhibition occurring through the loss of BLIMP-1. However, no difference in the proportion of PCs in the BM was observed between WM with and without 6q deletion. Furthermore, BLIMP-1 was not among downregulated genes in the present study, suggesting that loss of BLIMP-1 does not affect PC differentiation. Considering these results, the loss of BLIMP-1 did not appear to act as a loss of heterozygosity regarding $\mathrm{PC}$ differentiation.
We also observed IL21R and JAK3 overexpression in WM with del 6q. Investigators from the Mayo Clinic previously reported that the IL21/21R pathway contributes to IgM secretion and WM cell proliferation via the JAK/STAT signaling pathway in a WM cell line and WM patient samples [32]. Considering these results, it is conceivable that increased IgM levels in WM with 6q del are attributable to IL21/21R pathway activation. In the present cohort, the median M-protein level was higher in patients with $6 \mathrm{q}$ del than those without $6 \mathrm{q}$ del, although the result was not statistically significant because of the small sample size.

Our cDNA microarray analysis of WM with $6 \mathrm{q}$ del. also revealed the overexpression of FOXP1 and the Casitas Blineage lymphoma $\mathrm{b}$ gene (CBLB). FOXP1 overexpression is widely accepted to be a factor of poor prognosis in activated B-cell-like subtype diffuse large B-cell lymphoma and marginal zone B-cell lymphoma, mucosa-associated lymphoid tissue type $[33,34]$. Its overexpression was also shown to lead to the constitutive activation of the nuclear factor- $\kappa \mathrm{B}$ pathway as well as BCR, CD40, and TLR signaling pathways [35]. $C B L B$ encodes a protein involved in the ligand-induced clustering of BCR on the cell surface and delivery of BCRcaptured ligands to TLR9 [36]. Considering these findings, FOXP1 and CBLB might act as positive regulators for the BCR signaling pathway in WM with 6q del. patients (Figure 2).

Our present findings might represent an aggressive expression signature of WM with $6 \mathrm{q}$ del., although it should be noted that the study was rather exploratory and the sample size was very small. 


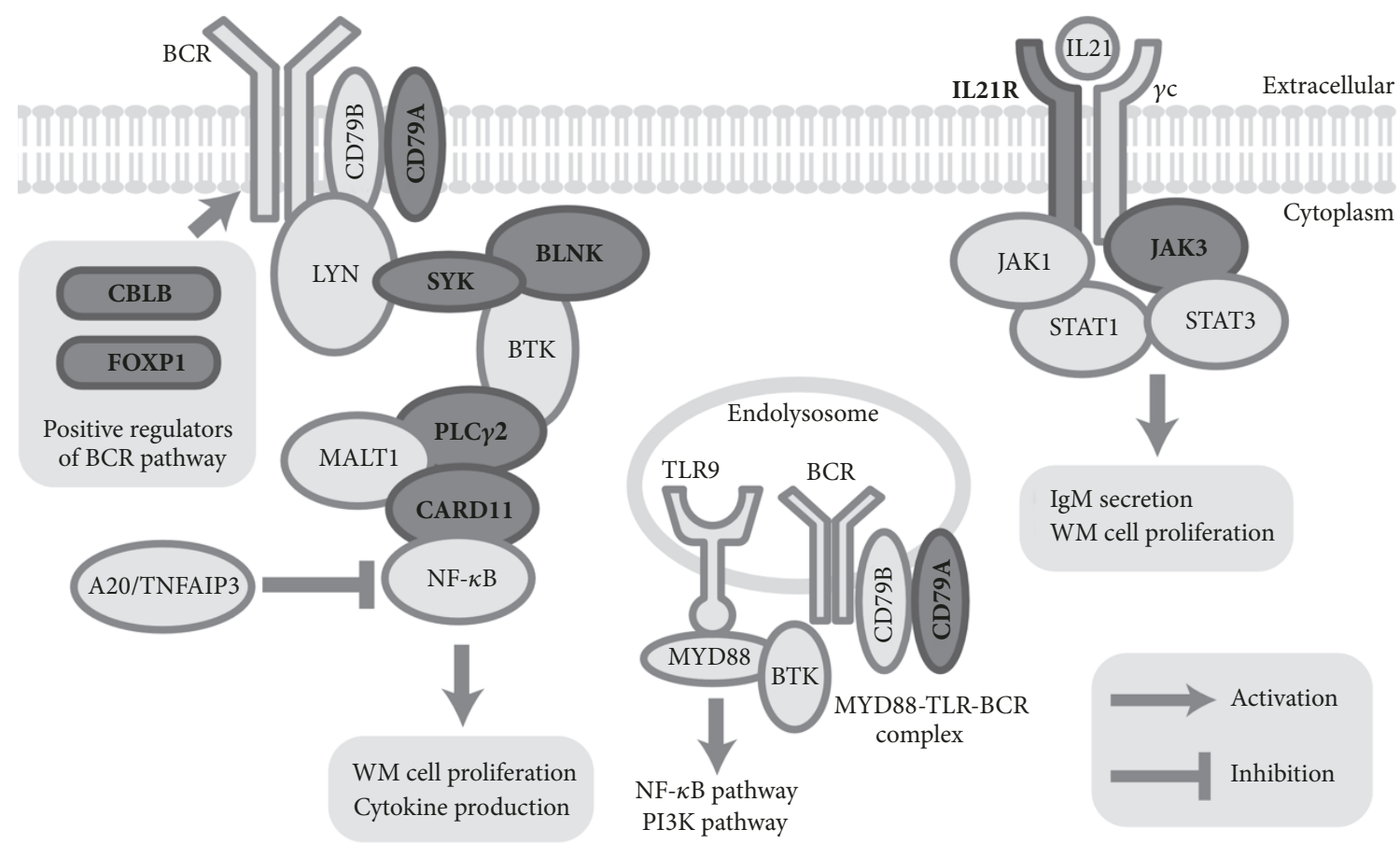

FIGURE 2: Schema of putative biological mechanisms of aggressiveness in Waldenström macroglobulinemia with 6q deletion. Upregulated genes in the present study are represented by bold font and dark shapes.

\section{Conclusion}

The present study suggested that the BCR signaling pathway and $I L 21 R$ expression are activated in WM with $6 \mathrm{q}$ del., and FOXP1 and CBLB appear to act as positive regulators of the BCR signaling pathway. Thus, our present findings might represent an aggressive expression signature of WM with $6 \mathrm{q}$ del.

\section{Data Availability}

The microarray data sets used in the present study were deposited in the Gene Expression Omnibus under accession number GSE70511 (http://www.ncbi.nlm.nih.gov/geo/query/ acc.cgi? acc $=$ GSE70511).

\section{Ethical Approval}

Investigation has been conducted in accordance with the ethical standards and according to the Declaration of Helsinki and according to national and international guidelines and has been approved by the authors' institutional review board. Institutional Review Board approval was obtained (Disaster Medical Center IRB-2014-8, National Cancer Center Hospital IRB-2013-142).

\section{Disclosure}

Masahiro Kiyota Current address is Internal Medicine, The University of Tokyo Hospital, Hongo, Bunkyo-ku, Tokyo 1138655, Japan.

\section{Conflicts of Interest}

The authors have no conflicts of interest to disclose.

\section{Authors' Contributions}

Naohiro Sekiguchi and Yukio Kobayashi participated in study design. Naohiro Sekiguchi performed Oligonucleotide microarray analysis. Akihisa Nagata, Naohiro Sekiguchi, Masahiro Kiyota, Ichiro Fukuda, Kazuaki Yamada, and Naoki Takezako prepared the tumor specimens and patient' information. Naohiro Sekiguchi and Yukio Kobayashi wrote the manuscript. All authors discussed the results and commented on the manuscript.

\section{Acknowledgments}

The authors would like to thank the members of the research group for useful discussions. This work was supported in part by Grants-in-Aid for Cancer Research from the Ministry of Health, Labour and Welfare of Japan (Clinical Cancer Research 22-014, 22-031, and 23-004), the National Cancer Center Research and Development Fund (23-A-17, 23-A-23, 26-A-4, 26-A-24, and 23-C-7), and a Japan Society for the Promotion of Science, Grant-in-Aid for Scientific Research (C) $(25461442,15 \mathrm{~K} 21647$, and 16K09865).

\section{Supplementary Materials}

Supplementary Table 1. Upregulated gene sets in WM with $6 \mathrm{q}$ del compared with WM without $6 \mathrm{q}$ del. The sheet contains 
428 probe sets. Among them, the first 342 probes that were allocated to genes or loci are shown according to Entrez Gene number in ascending order, and the remaining 86 probes that were not allocated to loci are shown according to fold changes in descending order. Supplementary Table 2. Downregulated gene sets in WM with 6q del compared with WM without $6 \mathrm{q}$ del. The sheet contains 112 probe sets. Among them, the first 98 probes that were allocated to genes or loci are shown according to Entrez Gene number in ascending order, and the remaining 14 probes that were not allocated to loci are shown according to fold changes in ascending order. (Supplementary Materials)

\section{References}

[1] S. H. Swerdlow, A. R. Cook Sohani Jr., S. A. Pileri et al. et al., "Lymphoplasmacytic lymphoma," in World health Organization Classification of Tumour of Hematopoietic and Lymphoid Tissues, S. H. Swerdlow, E. Campo, and N. L. Harris, Eds., IARC Press, Lyon, 2017.

[2] G. C. Issa, H. Leblebjian, A. M. Roccaro, and I. M. Ghobrial, "New insights into the pathogenesis and treatment of Waldenstrom macroglobulinemia," Current Opinion in Hematology, vol. 18, no. 4, pp. 260-265, 2011.

[3] A. Vijay and M. A. Gertz, "Waldenström macroglobulinemia," Blood, vol. 109, no. 12, pp. 5096-5103, 2007.

[4] F. Nguyen-Khac, J. Lambert, E. Chapiro et al., "Chromosomal aberrations and their prognostic value in a series of 174 untreated patients with Waldenström's macroglobulinemia," Haematologica, vol. 98, no. 4, pp. 649-654, 2013.

[5] H. Chang, C. Qi, Y. Trieu et al., "Prognostic relevance of 6q deletion in Waldenström's macroglobulinemia: A multicenter study," Clinical Lymphoma, Myeloma \& Leukemia, vol. 9, no. 1, pp. 36-38, 2009.

[6] E. M. Ocio, R. F. J. Schop, B. Gonzalez et al., "6q deletion in Waldenström macroglobulinemia is associated with features of adverse prognosis," British Journal of Haematology, vol. 136, no. 1, pp. 80-86, 2007.

[7] R. F. J. Schop, S. A. Van Wier, R. Xu et al., “6q deletion discriminates Waldenström macroglobulinemia from IgM monoclonal gammopathy of undetermined significance," Cancer Genetics and Cytogenetics, vol. 169, no. 2, pp. 150-153, 2006.

[8] E. Braggio, J. J. Keats, X. Leleu et al., "Identification of copy number abnormalities and inactivating mutations in two negative regulators of nuclear factor- $\kappa \mathrm{B}$ signaling pathways in Waldenström's macroglobulinemia," Cancer Research, vol. 69, no. 8, pp. 3579-3588, 2009.

[9] P. Morel, A. Duhamel, P. Gobbi et al., "International prognostic scoring system for Waldenström macroglobulinemia," Blood, vol. 113, no. 18, pp. 4163-4170, 2009.

[10] M. M. Oken, R. H. Creech, and D. C. Tormey, "Toxicity and response criteria of the Eastern Cooperative Oncology Group," American Journal of Clinical Oncology, vol. 5, no. 6, pp. 649-655, 1982.

[11] J. A. Kim, K. Im, S. N. Park, J. Kwon, Q. Choi et al., "MYD88 L265P Mutations Are Correlated with 6q Deletion in Korean Patients with Waldenström Macroglobulinemia," BioMed Research International, vol. 2014, Article ID 363540, 7 pages, 2014.
[12] J. McGowan-Jordan, A. Simons, and M. Schmid, An International System for Human Cytogenomic Nomenclature, Cytogenetic and Genome Research, 2016.

[13] C. Li and W. H. Wong, "Model-based analysis of oligonucleotide arrays: expression index computation and outlier detection," Proceedings of the National Acadamy of Sciences of the United States of America, vol. 98, no. 1, pp. 31-36, 2001.

[14] S. M. Ansell, R. A. Kyle, C. B. Reeder et al., "Diagnosis and management of waldenström macroglobulinemia: Mayo stratification of macroglobulinemia and risk-adapted therapy (mSMART) guidelines," Mayo Clinic Proceedings, vol. 85, no. 9, pp. 824-833, 2010.

[15] S. P. Treon, L. Xu, G. Yang et al., "MYD88 L265P somatic mutation in Waldenström's macroglobulinemia," The New England Journal of Medicine, vol. 367, no. 9, pp. 826-833, 2012.

[16] S. P. Treon and Z. R. Hunter, "A new era for Waldenstrom macroglobulinemia: MYD88 L265P," Blood, vol. 121, no. 22, pp. 4434-4436, 2013.

[17] M. Varettoni, L. Arcaini, S. Zibellini et al., "Prevalence and clinical significance of the MYD88 (L265P) somatic mutation in Waldenstrom's macroglobulinemia and related lymphoid neoplasms.", Blood, vol. 121, no. 13, pp. 2522-2528, 2013.

[18] G. Yang, Y. Zhou, X. Liu et al., "A mutation in MYD88 (L265P) supports the survival of lymphoplasmacytic cells by activation of Bruton tyrosine kinase in Waldenström macroglobulinemia," Blood, vol. 122, no. 7, pp. 1222-1232, 2013.

[19] S. L. Nutt, K. A. Fairfax, and A. Kallies, "BLIMP1 guides the fate of effector B and T cells," Nature Reviews Immunology, vol. 7, no. 12, pp. 923-927, 2007.

[20] J. Monge, E. Braggio, and S. M. Ansell, "Genetic Factors and Pathogenesis of Waldenström's Macroglobulinemia," Current Oncology Reports, vol. 15, no. 5, pp. 450-456, 2013.

[21] G. Bianchi, A. Sacco, S. Kumar, G. Rossi, I. Ghobrial, and A. Roccaro, "Candidate genes of Waldenström's macroglobulinemia: Current evidence and research," The Application of Clinical Genetics, vol. 6, pp. 32-42, 2013.

[22] M. Kato, M. Sanada, I. Kato et al., "Frequent inactivation of A20 in B-cell lymphomas," Nature, vol. 459, no. 7247, pp. 712-716, 2009.

[23] U. Novak, A. Rinaldi, I. Kwee et al., “The NF-kappaB negative regulator TNFAIP3 (A20) is inactivated by somatic mutations and genomic deletions in marginal zone lymphomas," Blood, vol. 113, no. 20, pp. 4918-4921, 2009.

[24] W. J. Chng, R. F. Schop, T. Price-Troska et al., "Gene-expression profiling of Waldenström macroglobulinemia reveals a phenotype more similar to chronic lymphocytic leukemia than multiple myeloma," Blood, vol. 108, no. 8, pp. 2755-2763, 2006.

[25] N. C. Gutiérrez, E. M. Ocio, J. de las Rivas et al., "Gene expression profiling of B lymphocytes and plasma cells from Waldenström's macroglobulinemia: Comparison with expression patterns of the same cell counterparts from chronic lymphocytic leukemia, multiple myeloma and normal individuals," Leukemia, vol. 21, no. 3, pp. 541-549, 2007.

[26] C. Jiménez, M. I. Prieto-Conde, M. García-Álvarez et al., "Unraveling the heterogeneity of IgM monoclonal gammopathies: a gene mutational and gene expression study," Annals of Hematology, vol. 97, no. 3, pp. 475-484, 2018.

[27] C. U. Niemann and A. Wiestner, "B-cell receptor signaling as a driver of lymphoma development and evolution," Seminars in Cancer Biology, vol. 23, no. 6, pp. 410-421, 2013. 
[28] K. V. Argyropoulos, R. Vogel, C. Ziegler et al., "Clonal B cells in Waldenström's macroglobulinemia exhibit functional features of chronic active B-cell receptor signaling," Leukemia, vol. 30, no. 5, pp. 1116-1125, 2016.

[29] A. L. Shaffer, K.-I. Lin, T. C. Kuo et al., "Blimp-1 orchestrates plasma cell differentiation by extinguishing the mature B cell gene expression program," Immunity, vol. 17, no. 1, pp. 51-62, 2002.

[30] A. L. Shaffer, A. Rosenwald, and L. M. Staudt, "Lymphoid malignancies: the dark side of B-cell differentiation," Nature Reviews Immunology, vol. 2, no. 12, pp. 920-932, 2002.

[31] J. D. Phelan, R. M. Young, D. E. Webster et al., "A multiprotein supercomplex controlling oncogenic signalling in lymphoma," Nature, vol. 560, no. 7718, pp. 387-391, 2018.

[32] L. S. Hodge, S. C. Ziesmer, Z. Z. Yang et al., "IL-21 in the bone marrow microenvironment contributes to IgM secretion and proliferation of malignant cells in Waldenstrom macroglobulinemia," Blood, vol. 120, no. 18, pp. 3774-3782, 2012.

[33] A. H. Banham, J. M. Connors, P. J. Brown et al., "Expression of the FOXP1 transcription factor is strongly associated with inferior survival in patients with diffuse large B-cell lymphoma," Clinical Cancer Research, vol. 11, no. 3, pp. 1065-1072, 2005.

[34] X. Sagaert, P. De Paepe, L. Libbrecht et al., "Forkhead box protein P1 expression in mucosa-associated lymphoid tissue lymphomas predicts poor prognosis and transformation to diffuse large B-cell lymphoma," Journal of Clinical Oncology, vol. 24, no. 16, pp. 2490-2497, 2006.

[35] M. van Keimpema, L. J. Grueneberg, and M. Mokry, "FOXP1 directly represses transcription of pro-apoptotic genes and cooperates with NF-kappaB to promote survival of human Bcells," Blood, vol. 124, no. 23, pp. 3431-3440, 2014.

[36] M. Veselits, A. Tanaka, S. Lipkowitz et al., "Recruitment of Cbl-b to $B$ cell antigen receptor couples antigen recognition to toll-like receptor 9 activation in late endosomes," PLOS ONE, vol. 9, no. 3, 2014. 


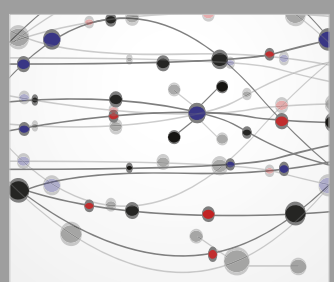

The Scientific World Journal
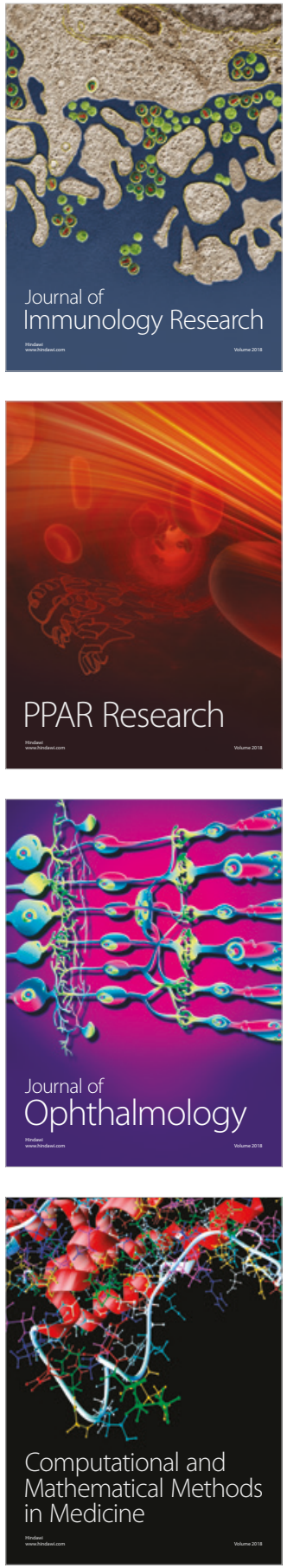

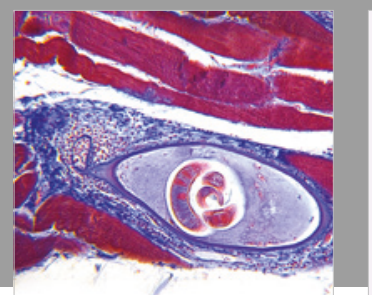

Gastroenterology Research and Practice

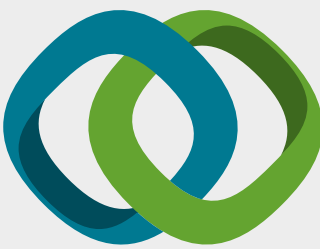

\section{Hindawi}

Submit your manuscripts at

www.hindawi.com
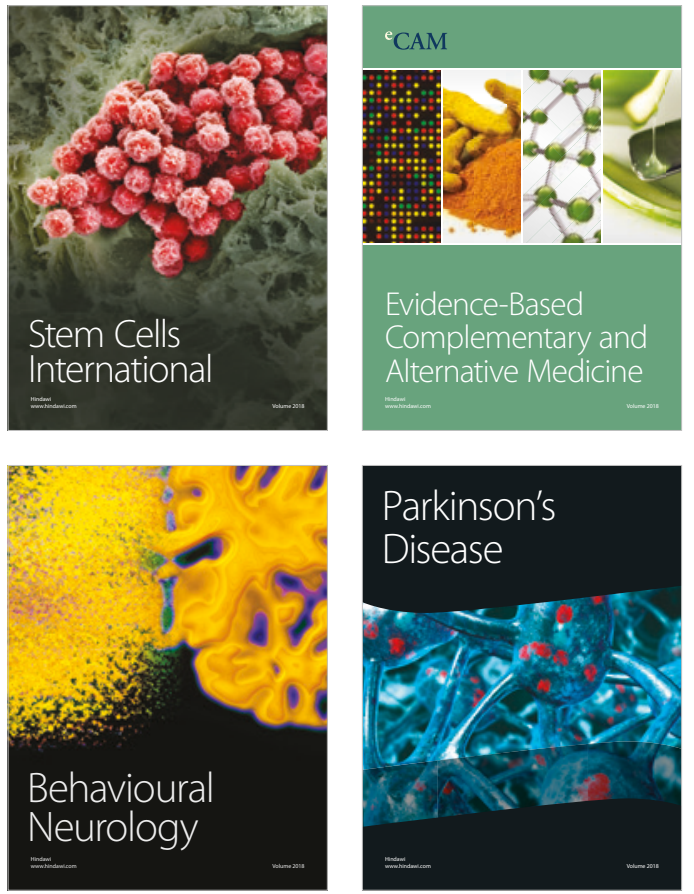

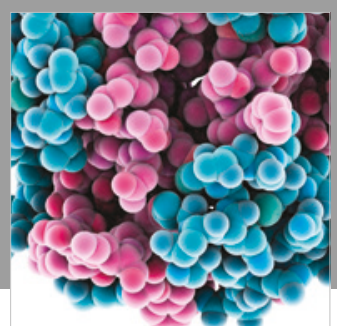

ournal of

Diabetes Research

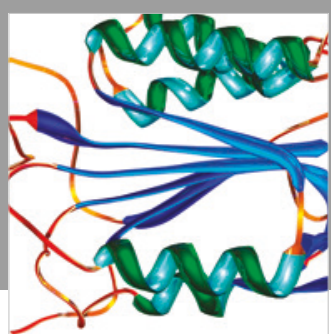

Disease Markers
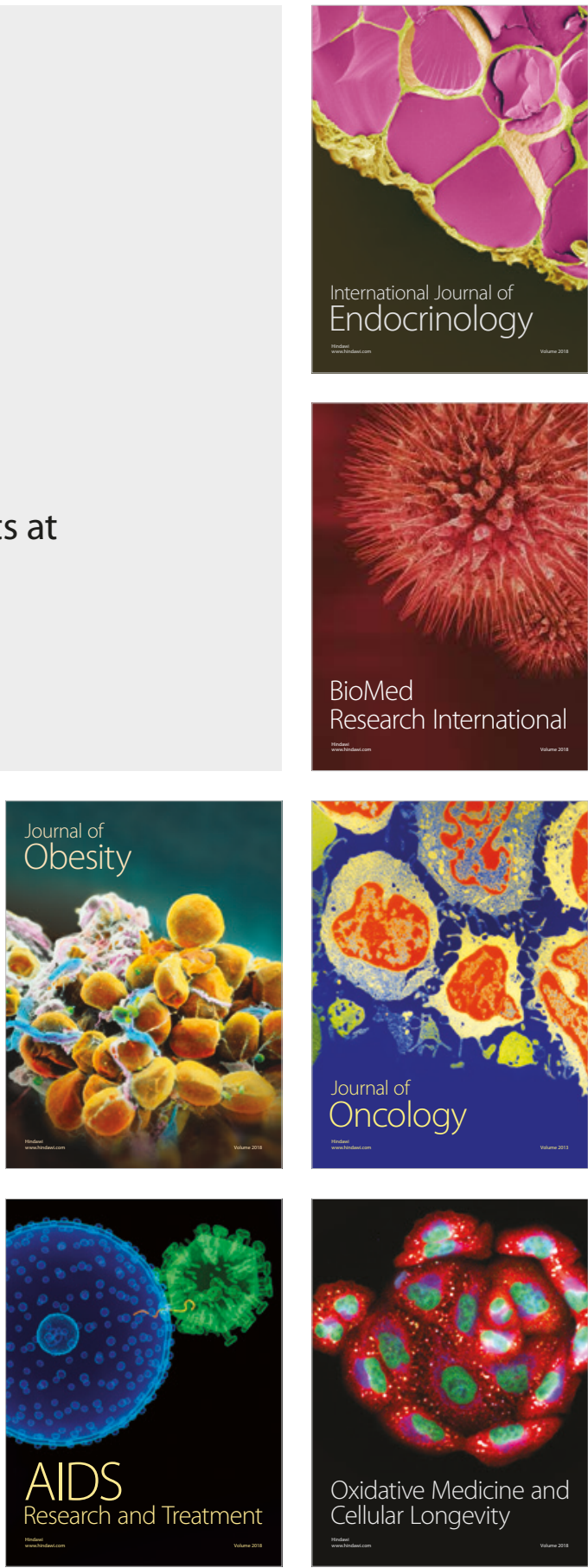